\title{
Small Bowel Intussusception due to Multiple Intestinal Metastases from Lung Adenocarcinoma
}

\author{
Faten Limaiem, Saadia Bouraoui \\ Tunis Faculty of Medicine, University of Tunis El Manar, Tunis, Tunisia
}

Correspondence to: Dr. Faten Limaiem; email: fatenlimaiem@yahoo.fr

Received: 1 May 2020; Revised: 9 Oct 2020; Accepted: 07 Nov 2020; Available online: 10 Mar 2021

\begin{abstract}
Summary
Metastatic small bowel tumors are rare. They often present with small bowel occlusion, bleeding, perforation, or intestinal intussusception. Pulmonary adenocarcinoma with metastasis to the small intestine causing intussusception is exceedingly rare. A 72-yearold male patient with a past medical history of left lung adenocarcinoma, presented to the emergency department with abdominal pain and vomiting. On admission, an X-ray of the abdomen without preparation showed some hydroaeric levels on the small intestine. The abdominopelvic computed tomography (CT) scan revealed an ileo-ileal invagination, with an image of the invagination rod. The patient underwent a surgical resection of the small bowel with the removal of the ischemic areas and immediate anastomosis. Macroscopic examination of the surgical specimen revealed five tumor masses varying in size between 1 and $4 \mathrm{~cm}$. The histological examination of the samples taken from these tumors, coupled with an
\end{abstract}

immunohistochemical study confirmed the diagnosis of intestinal metastases of pulmonary origin expressing cytokeratin 7 (CK7) and thyroid transcription factor 1 (TTF1) but negative for CK20, chromogranin, and synaptophysin. The postoperative course was uneventful, and the symptoms transiently recovered. However, the patient died 3 months later. We should consider multiple metastatic lesions in adult intussusception, especially in patients with a past medical history of lung cancer.

Keywords: Intussusception, lung adenocarcinoma, intestinal metastasis

Ann Afr Surg. 2021; 18(3): 189-193

DOI: http://dx.doi.org/10.4314/aas.v18i3.12

Conflicts of Interest: None

Funding: None

(C) 2021 Author. This work is licensed under the Creative Commons Attribution 4.0 International License.

\section{Introduction}

Intussusception is a frequent etiology of small bowel obstruction in the pediatric age group compared to the elderly patients. Adult intussusception represents $5 \%$ of all intussusception cases. It is the cause of only $1 \%$ of all adult bowel obstruction cases. The majority of cases in adults are due to definable structural lesions (1). However, most lesions are benign and malignancymediated intussusception is rare (2). Cases of lung adenocarcinoma with metastases to the small intestine causing intussusception are very rare with only 42 cases reported in the literature (3). Herein, the authors report a new case of an unexpected small bowel intussusception due to multiple metastatic pulmonary adenocarcinomas.

\section{Case report}

A 72-year-old male patient with a past medical history of left lung adenocarcinoma, presented to the emergency department with abdominal pain and vomiting. He described the absence of gas and feces for 2 days. On admission, the patient was afebrile. The clinical examination showed a slightly sensitive and distended abdomen with increased tympanic sound. There was no rebound tenderness, hepatosplenomegaly, or abdominal 
mass on palpation. The digital rectal exam was normal. Laboratory studies were within normal limits.

The X-ray of the abdomen without preparation showed some hydroaeric levels on the small intestine (Figure 1A). Abdominal sonography revealed a "pseudo kidney sign" at longitudinal view and a "donut sign" at axial view (Figure 1B). The abdominopelvic computed tomography (CT) scan revealed an ileo-ileal invagination (Figure 1C), with an image of the invagination rod. The patient underwent surgical resection of the small bowel with removal of the ischemic areas, and immediate anastomosis. An exploratory laparotomy performed under the diagnosis of an organic small bowel obstruction revealed ileo-ileal intussusception with signs of bowel ischemia. A moderate amount of clear ascites was noted without evidence of peritoneal carcinomatosis or gross mesenteric lymph node enlargement. During emergency surgery, the patient underwent segmental resection of the region of the small bowel showing intussusception with removal of the ischemic areas, and immediate anastomosis. Macroscopic examination of the surgical specimen revealed five tumor masses varying in size between 1 and $4 \mathrm{~cm}$. The histological examination of the samples taken from these tumors, coupled with an immunohistochemical study confirmed the diagnosis of intestinal metastases of a poorly differentiated adenocarcinoma (Figure 2A). Tumor cells showed marked nuclear atypia (Figure 2B) and several mitotic figures. Immunohistochemical study revealed positive immunostaining of tumor cells for cytokeratin 7 (CK7) (Figure 3A) and thyroid transcription factor 1 (TTF1) (Figure 3B), thus confirming a pulmonary origin of the intestinal metastases. The tumor cells were negative for CK20, synaptophysin, and chromogranin. Based on these findings, a diagnosis of ileo-ileal intussusception due to small bowel metastasis of pulmonary adenocarcinoma was made. The post-operative course was uneventful, and the symptoms transiently recovered. Chemotherapy was not administered due to the patient's poor general condition and performance status. The patient died after 3 months of follow-up.

\section{Discussion}

Intussusception is the telescoping of a proximal segment of the gastrointestinal tract into the lumen of the contiguous distal segment of the gastrointestinal tract (4). The exact mechanism of intussusception is undetermined. However, any tumor in the intestinal wall or irritant within the lumen that changes the normal peristaltic activity can result in an invagination. Intussusception in children is often primary; there are no pathologic lead points in the majority of children with intussusception (4). Approximately $90 \%$ of adult cases of intussusception are due to a pathologic condition that serves as a lead point (4-6). The etiologies of small bowel intussusceptions include benign lesions, namely Meckel's diverticula, hamartomatous polyps, and strictures. Malignancy accounts for 6 to $30 \%$ of cases of small bowel intussusceptions in adults (2). These malignancies comprise primary lesions and metastases, in particular from the lung, breast, and malignant melanoma. The metastatic tumors are more frequent than primary malignancies. Pulmonary cancer is the predominant etiology of cancer death worldwide. The most common metastatic sites from lung cancer include the bones, the brain, the adrenal glands, and the liver. Intestinal metastases are, however, exceedingly rare (7). Pulmonary large cell carcinoma is the most frequent histological subtype that metastasizes to the gastrointestinal tract. Nevertheless, the clinical manifestations do not differ by the histologic type. Intestinal metastasis from primary lung cancer is associated with a poor prognosis $(3,8)$. The patients usually present with nonspecific symptoms of bowel obstruction, namely abdominal pain, abdominal distension, nausea and vomiting $(3,9)$. On physical examination, patients may show localized or diffuse abdominal tenderness and pressure pain, but often symptoms are non-specific, and the clinical presentation is inconspicuous (3). In our case, physical examination showed a slightly sensitive and distended abdomen with tympanism but without a palpable mass. The precise preoperative diagnosis of intussusception may be very difficult. The reported preoperative diagnosis rates are about $50 \%$ (3). 


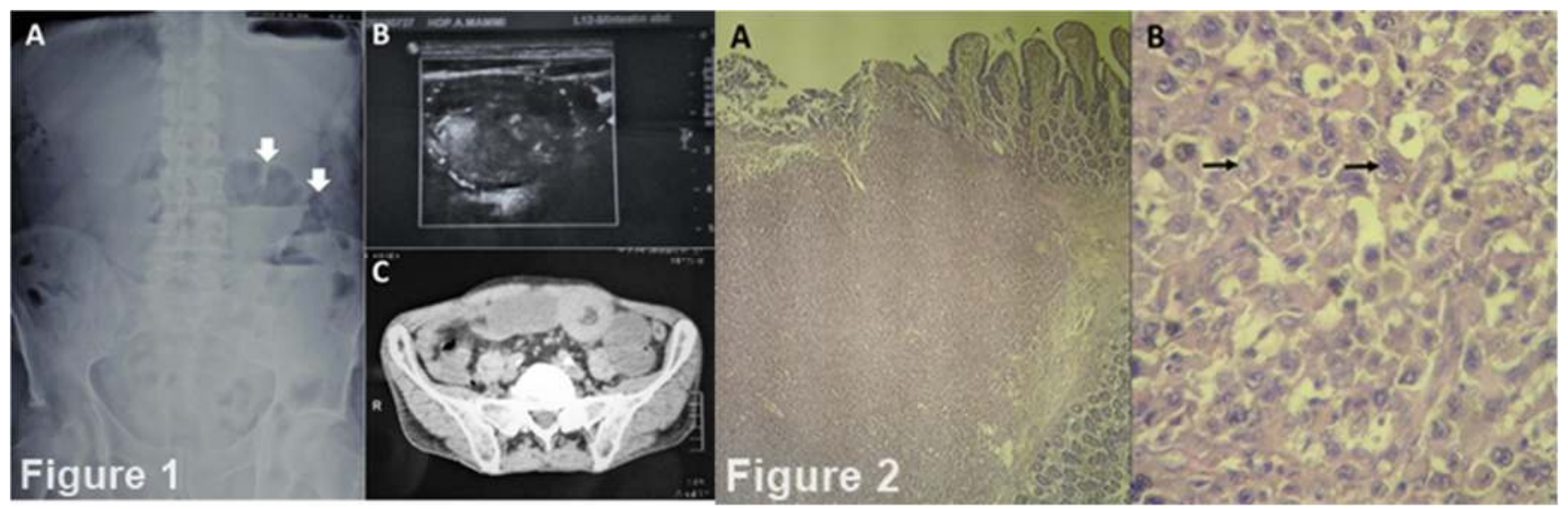

Figure 1. (A) X-ray of the abdomen without preparation showing some hydroaeric levels on the small intestine (white arrows). (B) Abdominal ultrasonography revealing increased intestinal wall thickness with a target sign in the right lower quadrant. (C) Abdominal CT scan demonstrating dilated intestinal loops with the characteristic target shaped appearance, highly suggestive of enteric intussusception in the ileum. Figure 2: (A) Histologic analysis identified a poorly differentiated adenocarcinoma proliferating mainly in the submucosa and muscular layer and invading the subserosa (hematoxylin and eosin staining, $\times 40$ magnification). (B) Tumor cells showing marked atypia (hematoxylin and eosin staining, $\times 400$ magnification) (arrows).

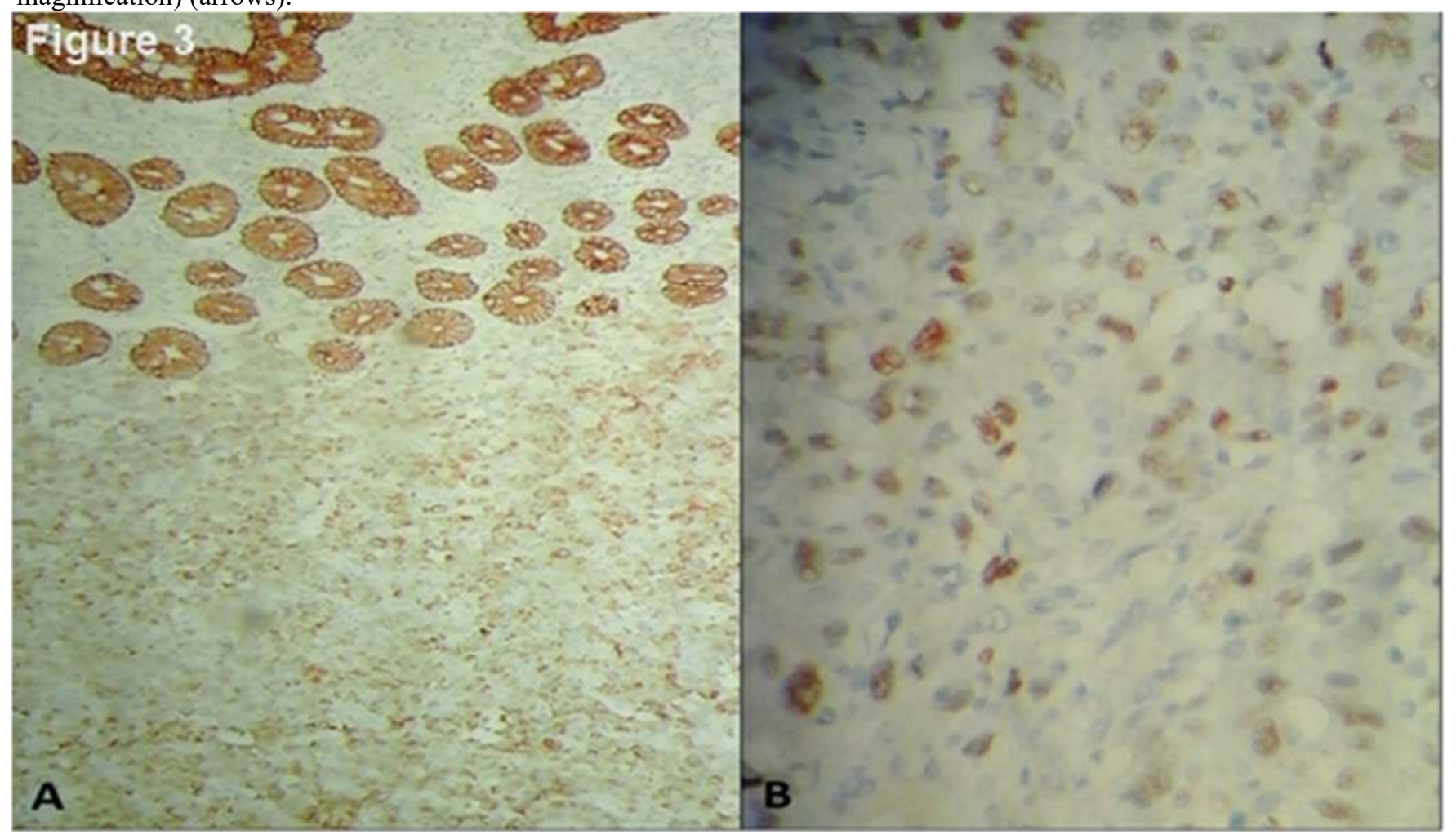

Figure 3. (A) Immunohistochemical staining of the tumor cells showing diffuse positivity for cytokeratin $(\times 200$ magnification). (B) Immunohistochemical staining of the tumor cells showing diffuse positivity for TTF1 $(\times 200$ magnification). 
Although imaging studies can be useful in identifying intussusception, initial diagnosis is still often delayed or missed and is established only at surgical exploration (3, 10, 11). Computed tomography scan is the most sensitive imaging technique to confirm intussusception. Moreover, a CT scan can define the site and the nature of the mass and its relationship with the surrounding tissues. It may also help in the staging of patients with suspected malignancy causing the intussusception (3). In the present case, ultrasonography and the CT scan revealed the characteristic signs of intussusception and were useful in diagnosis. Immunohistochemistry is the only reliable tool to establish the diagnosis of metastatic small bowel tumors. Primary or metastatic pulmonary adenocarcinoma usually shows positive immunostaining for CK7, TTF1, and Napsin A. The latter has a sensitivity and a specificity of $92 \%$ and $100 \%$ in the primary lung adenocarcinoma, whereas TTF- 1 has a sensitivity and a specificity of $84.5 \%$ and $96.4 \%$ in primary lung adenocarcinoma.

In our case, histological examination revealed a poorly differentiated adenocarcinoma that showed positive immunostaining for CK7 and TTF1 but was negative for chromogranin A and synaptophysin. Napsin A was not performed, as our laboratory lacked this antibody.

Despite the poor prognosis of these patients, most researchers agree that surgical treatment is required as long as the patient's physical condition can tolerate the procedure. On the other hand, a literature review showed no survival benefit for patients receiving adjuvant chemotherapy, radiotherapy, or targeted therapy (10, 11).

\section{Conclusion}

In conclusion, it is important to keep in mind that although rare, small bowel metastases of lung cancers can occur regardless of their histological type and may cause intussusception (4). We should therefore consider the presence of pulmonary malignancies in the history of any patient presenting with abdominal symptoms (4).

\section{Informed consent}

Written informed consent was obtained from the patient.

\section{References}

1. Di JZ, Peng JY, Wang ZG. Prevalence, clinicopathological characteristics, treatment, and prognosis of intestinal metastasis of primary lung cancer: a comprehensive review. Surg Oncol. 2014; 23:72-80.

2. Begos DG, Sandor A, Modlin IM. The diagnosis and management of adult intussusception. Am J Surg. 1997; 173(2): 88-94.

3. Otera H, Ikeda F, Nakagawa $\mathrm{S}$, et al. Intussusception of small intestine due to metastasis of large cell carcinoma of the lung with a rhabdoid phenotype. Eur Respir Rev. 2010 ; 19: 248-252.

4. Marinis A, Yiallourou A, Samanides L, et al. Intussusception of the bowel in adults: a review. World $\mathrm{J}$ Gastroenterol. 2009; 15(4): 407-411.

5. Alabbas $Z$, Issa $M$, Omran $A$, et al. Mesenteric inflammatory myofibroblastic tumor as a rare cause of small intestinal intussusception. J Surg Case Rep. 2020; 23(9): rjaa322.

6. Di Buono G, Randisi B, Romano G, et al. Recurrent intussusception of small bowel in a young patient due to metastases from cardiac undifferentiated pleomorphic sarcoma: A first ever case report. Int J Surg Case Rep. 2020; 14: S2210-2612(20)30738-0.

7. Guner A, Karyagar S, Livaoglu A. Small bowel intussusception due to metastasized sarcomatoid carcinoma of the lung: A rare cause of intestinal obstruction in adults. Case Rep Surg. 2012; 2012:962683.

8. Rossi G, Marchioni A, Romagnani E, et al. Primary lung cancer presenting with gastrointestinal tract involvement: clinicopathologic and immunohistochemical features in a series of 18 consecutive cases. J Thorac Oncol. 2007; 2(2): 115-120.

9. Pollheimer MJ, Eberl T, Baumgartner K, et al. Ileocecal intussusception caused by lung cancer metastasis. Wien Klin Wochenschr. 2009; 121: 413-416.

10. Kagohashi K, Kadono K, Satoh H, et al. Intussusception due to intestinal metastasis from lung cancer. Lung Cancer. 2007; 57:247-248.

11. Lee PC, Lo C, Lin MT, et al. Role of surgical intervention in managing gastrointestinal metastases from lung cancer. World J Gastroenterol. 2011; 17: 4314-4320. 